\title{
Kirgistan, Land wo die Berge den Himmel berühren \\ Thomas Ster
}

\begin{abstract}
In July 2015, the author organized a field trip to Kyrgyzstan. Some of the plants observed on the tour are listed. Beauty of the landscape contrasts with the environmental destruction caused by the construction of gold mines.
\end{abstract}

\section{Zusammenfassung}

Im Juli 2015 fand eine Exkursion nach Kirgistan statt. Der Autor war Exkursionsleiter und stellte die Routen zusammen. Das stark gebirgige zentralasiatische Land wird kurz vorgestellt, landschaftliche Gliederung und klimatische Bedingungen werden erläutert. Es wurden auch die alten Walnuss-Wälder besucht. Einige der auf der Tour beobachteten Pflanzen werden aufgelistet. Die landschaftliche Schönheit und Schutzwürdigkeit stehen in Kontrast zur Umweltzerstörung durch den Goldabbau.

\section{Exkursion nach Kirgistan}

Beeindruckt von den Erlebnissen im benachbarten Kasachstan anlässlich einer Exkursion 2013 organisierte die Österreichische Gartenbaugesellschaft, Landesgruppe Steiermark, vom 2. Juli bis 21. Juli 2015 eine botanische Exkursion nach Kirgistan. Die örtliche Organisation und Begleitung erfolgte auch diesmal durch Stephan Flechtner und Dagmar Schreiber. Botanisch betreut wurden wir von Professor Georgij Laskov und Almagül Kenderboeva, Akademie der Wissenschaften in Bishkek. Ein ausführlicher Bericht ist beim Autor hinterlegt.

\section{Kirgistan, das Land, wo die Berge den Himmel berühren}

Während Nepal am Fuße des Himalayas als Land am Dach der Welt bekannt geworden ist, gibt sich

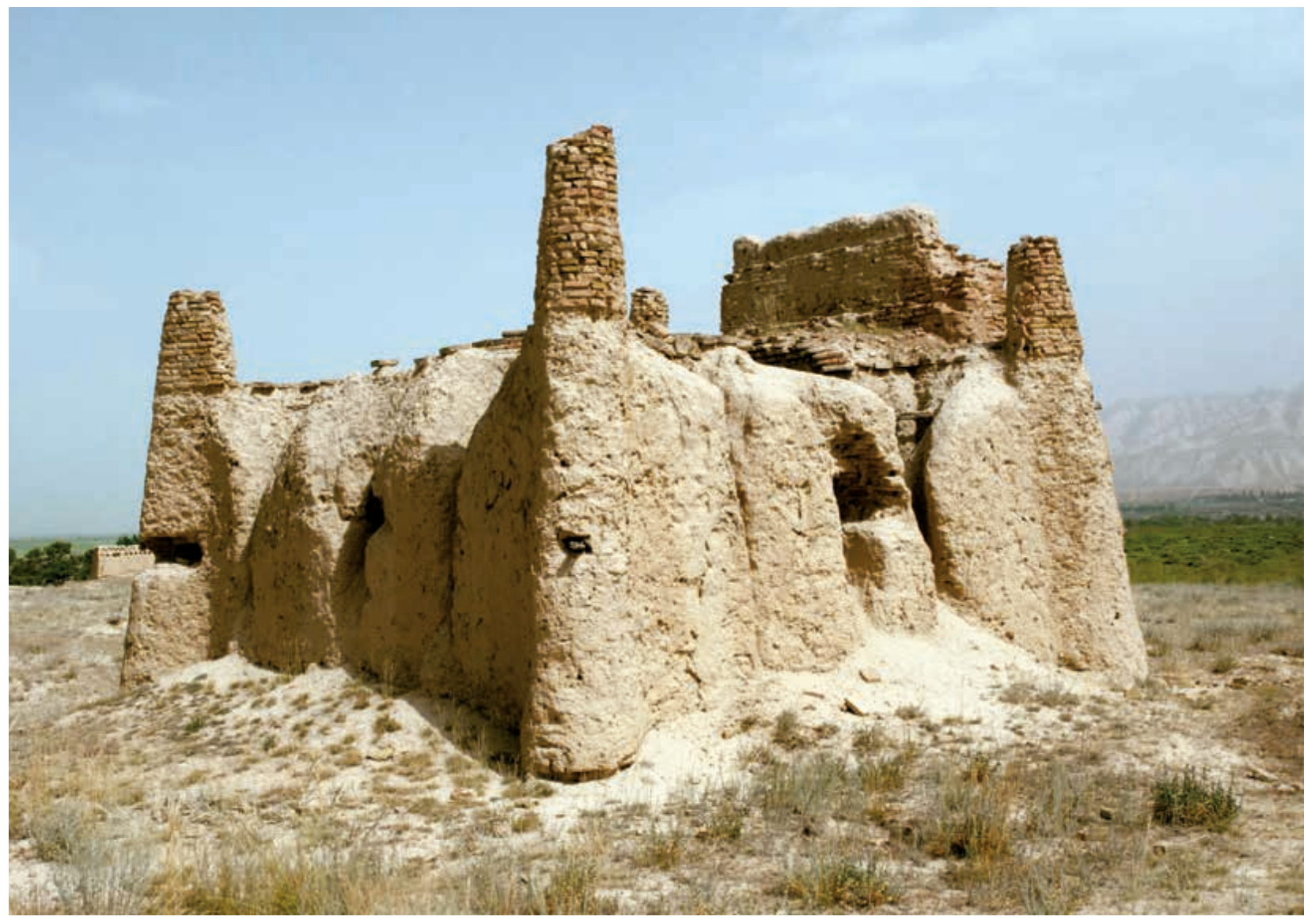

Abb. 1: Historische Karawanserei am Rande der Seidenstraße. 


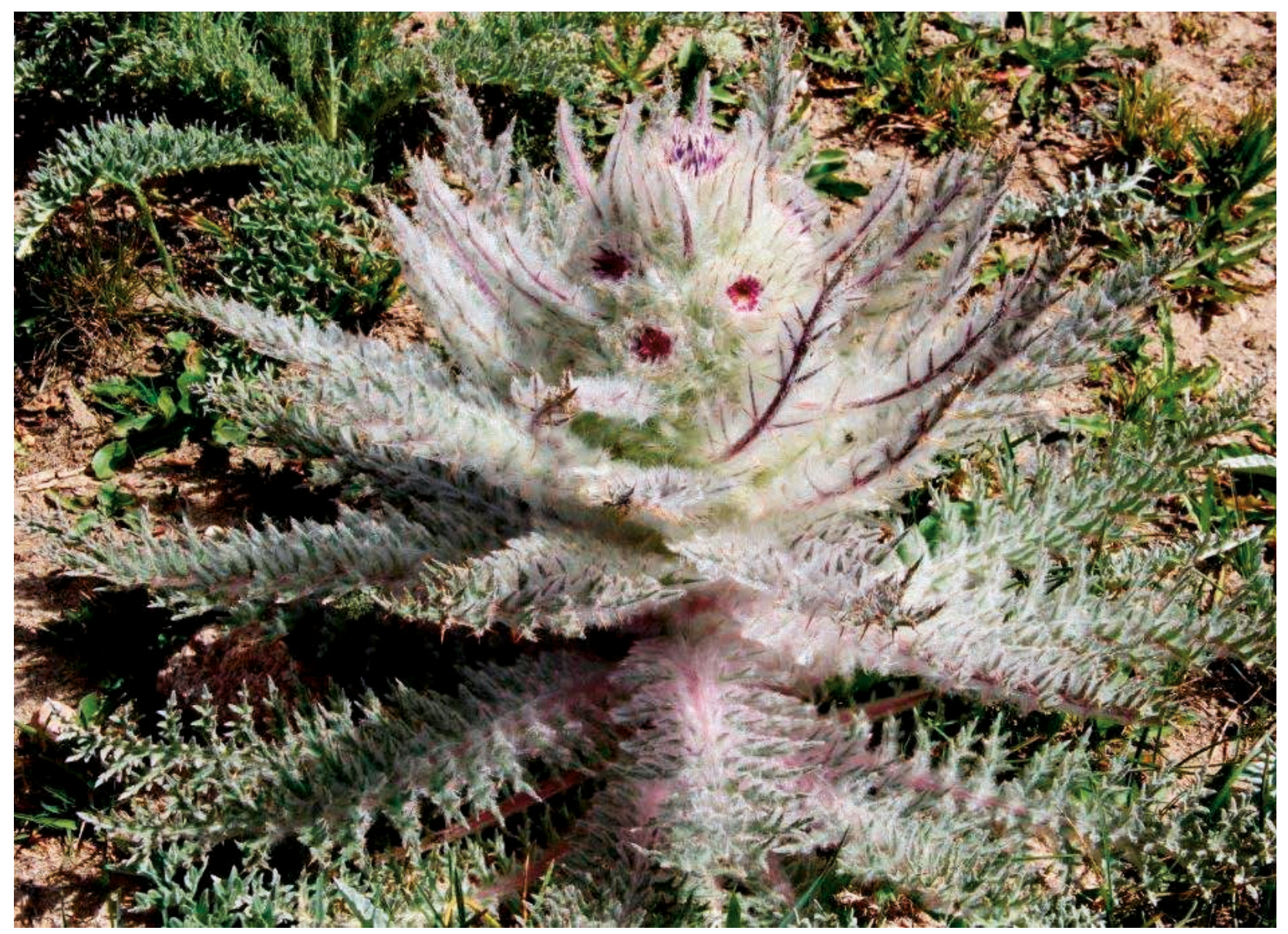

Abb. 2: Schmalhausenia nidulans.

Kirgistan viel bescheidener. Obwohl die mächtigen Bergriesen des Tien Shan und die Ausläufer des Pamir-Gebirges nicht weniger eindrucksvoll dieses kleine zentralasiatische Land beherrschen, gilt es als Land wo die Berge den Himmel berühren. Mehr als $70 \%$ des Landes liegen über $3000 \mathrm{~m}$. Am Rande der historischen Seidenstraße gelegen, mehrfach erobert und zerstört, wurde es 1876 mit großen Teilen des heutigen Usbekistan (Kokander Kahnat) dem Zarenreich angegliedert und später Teil der UdSSR. Von den Verfolgungen und Verwerfungen während der STALIN-Diktatur und der großen Not während des Krieges, aber auch von landschaftlicher Schönheit, berührenden menschlichen Schicksalen und neuzeitlichen Bedrohungen erzählen die Romane des wohl berühmtesten Literaten des Landes, Tschingis Achmatov.

Mit dem Zerfall der UdSSR erlangte Kirgistan 1991 zwar die Unabhängigkeit, jedoch flammten immer wieder ethnische Auseinandersetzungen auf, vor allem mit der usbekischen Minderheit im
Südwesten des Landes um die zweitgrößte Stadt Osh. Von der westlichen Staatengemeinschaft fast vergessen und enttäuscht, ringt das bitterarme Land um eine Zukunft, die nicht nur in der einzigen Goldmine in dem an Bodenschätzen armen Land liegt, sondern auch in einem sanften Tourismus. Die landschaftliche Schönheit in den einsamen Gebirgsregionen, das kristallklare, leicht salzige Wasser des Ysyk Kul, dem zweitgrößten Gebirgssee der Erde, der durch warme unterirdische Quellen auch im Winter nicht zufriert und nicht zuletzt eine kaum zu übertreffende Gastfreundschaft wären gute Voraussetzungen.

\section{Landschaftliche Gliederung}

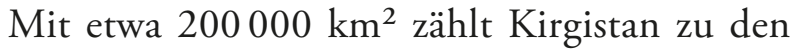
kleinsten zentralasiatischen Ländern. Das stark gegliederte, durch Auffaltung entstandene Gebirgsrelief des Tien Shan beherrscht den Großteil des Landes und erreicht im Osten an der Chinesischen Grenze mit dem 7439 m hohen Pik Pobedy (Dschengisch Tschokusu) den höchsten 


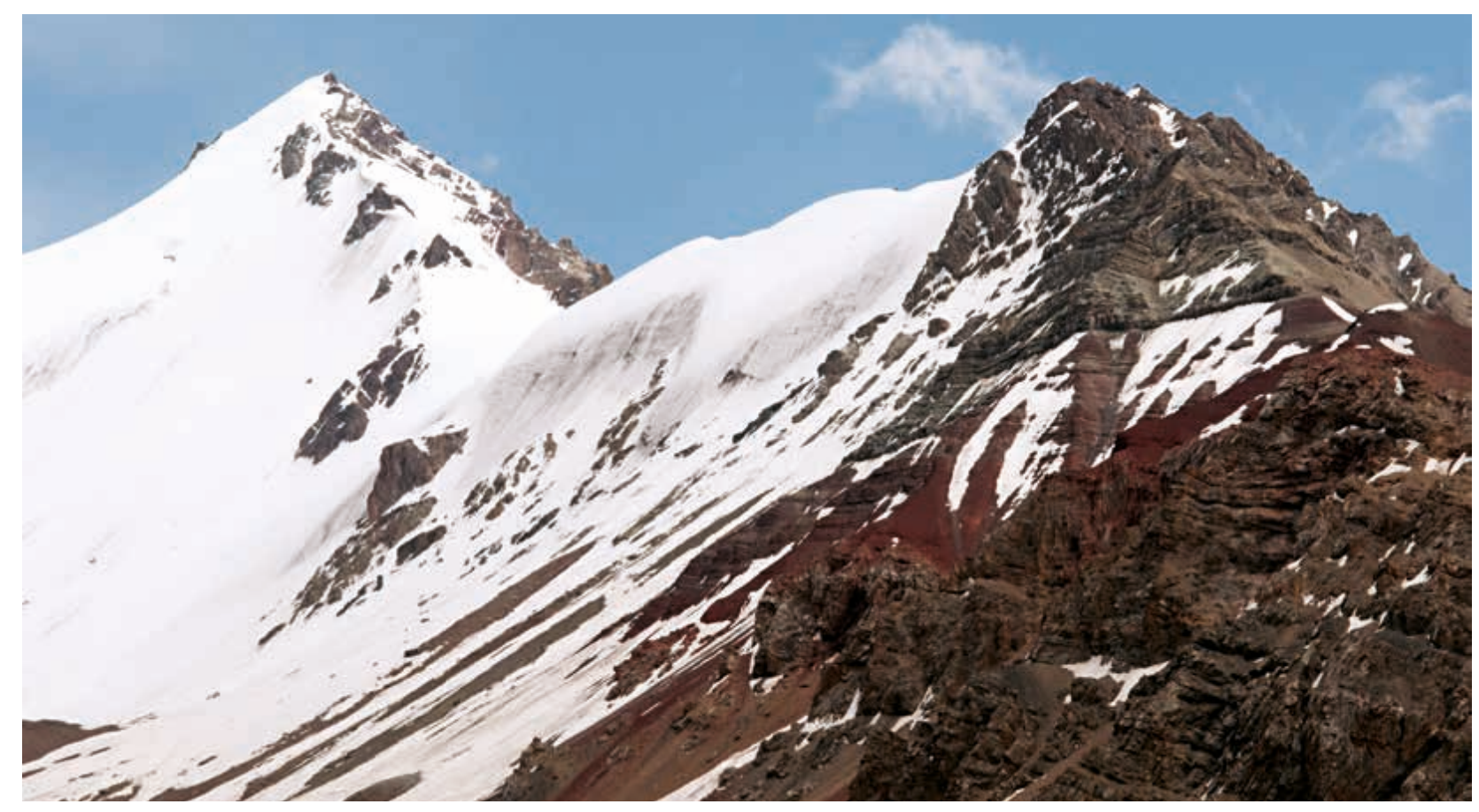

Abb. 3: Pik Lenin, $7134 \mathrm{~m}$ hoch.

Berg Kirgistans. Ganz im Westen, im Grenzgebiet zu Tadschikistan, liegen die nördlichen Ausläufer des Pamir mit der Transalai-Kette und dem Pik Lenin (7 $134 \mathrm{~m}$ ). Die mächtigen Gebirgsketten schirmen das Land weitgehend von den aus Süden herandrängenden Niederschlägen $\mathrm{ab}$, sodass weitläufige Steppengebiete und Halbwüsten vorherrschen. Dennoch ist das Land durch die zahlreichen Gletscher und Seen wasserreich. Der Naryn, größter Fluss des Landes, durchzieht das fruchtbare Fergana Becken, wird nach dem Zusammenfluss mit dem Kara Daryja in Usbekistan zum Syrdaria und mündet nach $2200 \mathrm{~km}$ in den nördlichen Aralsee, der durch verschiedene Maßnahmen vor der gänzlichen Austrocknung bewahrt worden ist. Auf nur 7 \% des Landes, im Fergana Becken an der Grenze zu Usbekistan, ist Ackerbau möglich. Hier wird der berühmte rosakörnige Reis neben Baumwolle und Wassermelonen angebaut.

\section{Die Walnusswälder im}

\section{Biosphärenreservat Sary Tschelek}

Stolz sind die Kirgisen auf die wenigen Waldgebiete, insbesondere auf das Biosphärenreservat Sary Tschelek mit den letzten Walnusswäldern. Die Bergketten des Tien Shan verlaufen fast ausschließlich in West-Ost Richtung. Das Fergana-Becken wird im Osten durch das Tschatkal-Gebirge be- grenzt, das in Nord-Süd Richtung verläuft und damit die vom Westen herankommenden Regenwolken aufhält. $1200 \mathrm{~mm}$ Niederschlag ermöglichen eine für Kirgistan einzigartige Waldgesellschaft, ein „Schatzkästchen“. Hier haben 113 Baum- und Straucharten, 150 Vogelarten, 21 Säugetiere und über 3000 Insekten, davon viele endemisch, ein letztes Rückzugsgebiet gefunden. Nur durchsetzt von Wildobst wie Äpfeln (Malus sieversii) und Wild-Pflaumen-Arten (Prunus sogdiana) ist der Walnusswald mit bis zu 250-jährigen Baumrie-

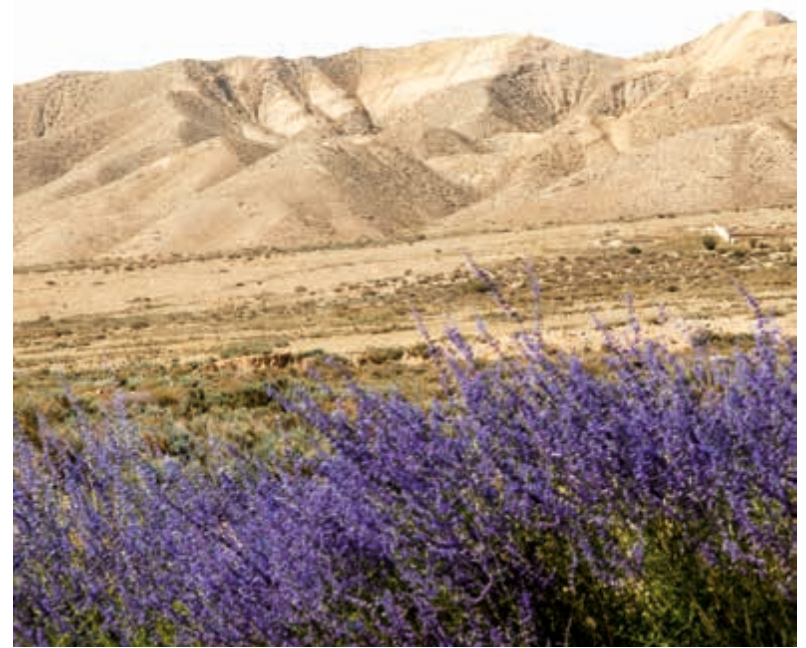

Abb. 4: Steppengebiete und Halbwüsten sind in Kirgistan landschaftsprägend. Im Vordergrund Perovskia angustifolia. 
sen das Herzstück des Schutzgebietes. Die erlaubte Frucht- und Weidenutzung ist allerdings derart intensiv, dass nur alte Nussbäume vorhanden sind und der notwendige Jungwuchs vollkommen fehlt.

Um den Sary Tschelek-See, wo Abies sibirica subsp. semenovii-Bäume bis zum Seeufer reichen, ist der Gehölzunterwuchs besonders artenreich: Abelia corymbosa, Exochorda tianschanica, Acer turkestanicum und die erst vor wenigen Jahren entdeckte Aflatunia (Prunus) ulmifolia, die zwischen Mandel und Pflaume steht.

Beeindruckend sind die von Doldenblütlern dominierten Bergwiesen, wo u. a. die bis zu $3 \mathrm{~m}$ hohe Ferula inciso-serrata wächst. Im Frühjahr muss es eine wahre Farbenpracht sein, wenn die karmesinroten Pfingstrosen (Paeonia hybrida) mit Tulipa zennaidae, Allium oreophilum und anderen Geophyten blühen.

\section{Artschawälder und Tien Shan-Fichten}

Verstreut im östlichen Landesteil kommen die Wacholder- oder Artschawälder vor. Sehr lockere Baumbestände aus drei Wacholder-Arten (Juniperus seravschanica, J. semiglobosum und J. turkestanica). Juniperus turkestanica bildet in Westkirgistan die Baumgrenze und kann bis über $3500 \mathrm{~m}$ steigen. Die Holznutzung aller Wacholder-Arten ist weitgehend eingestellt.

Die fast ausschließlich von der Tien Shan-Fichte Picea schrenkiana (mit den Unterarten schrenkiana und tianschanica) aufgebauten Wälder sind auf das Tien Shan-Gebirge und einige isolierte Gebiete im Pamir beschränkt. Auf Grund des extremen Holzmangels wurde der Waldbestand mittlerweile bis auf wenige Reste vernichtet. Dank nachhaltig wirkender Schutzmaßnahmen konnten die letzten Bestände der bis zu $60 \mathrm{~m}$ hoch werdenden Tien Shan-Fichte erhalten werden. Der schlanke, säulenförmige Wuchs erinnert an die Serbische Fichte.

\section{Die Sommerweide am Song Kul}

Nirgendwo erlebt man Kirgistan in seiner Ursprünglichkeit deutlicher als auf der Hochebene um den Song Kul. In den Sommermonaten, wenn in den Tälern Dürre herrscht, ziehen die Kirgisen

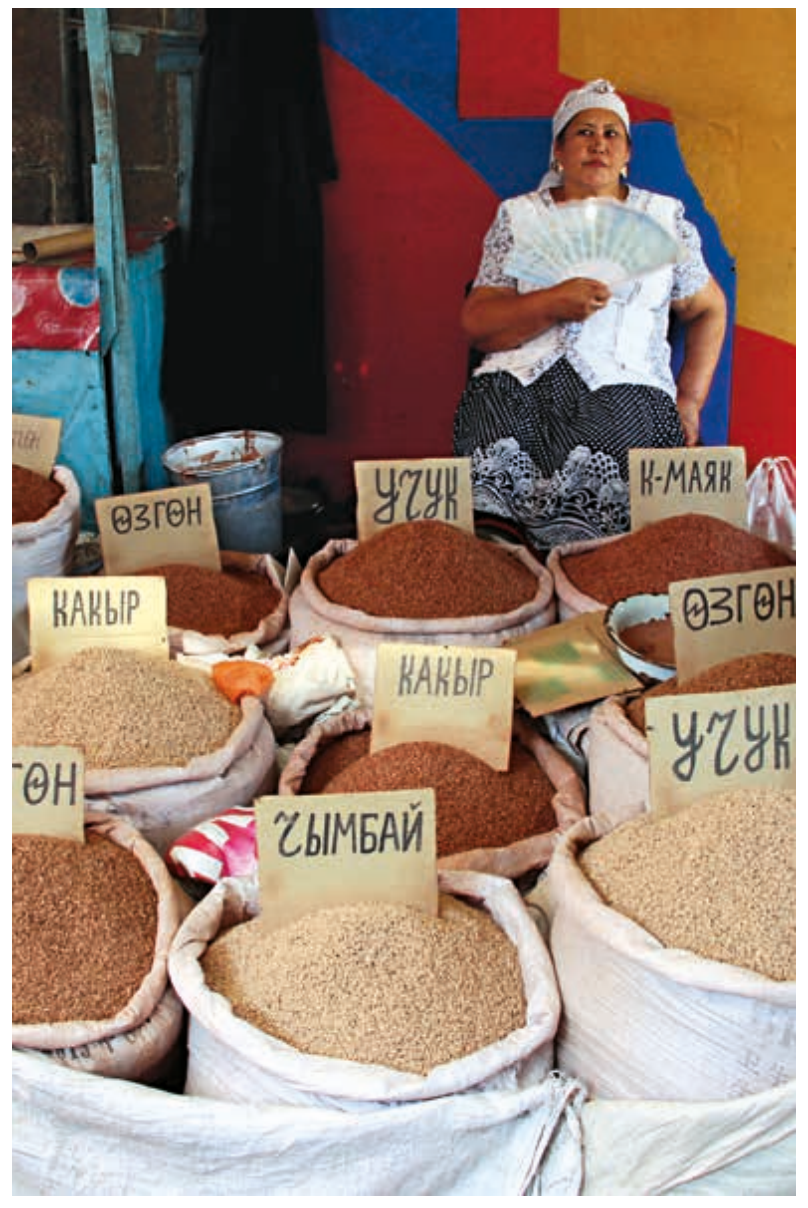

Abb. 5: Der berühmte rosakörnige Reis auf einem lokalen Markt.

mit ihren Viehherden auf die grünen Weidegründe in höhere Bergregionen. Die Jurtensiedlungen ergeben ein fast archaisches Bild und erinnern an die Jahrhunderte alte Nomadentradition. Wie in vielen anderen Landesteilen ist die starke Überweidung ein Problem geworden und durch den Klimawandel bleiben auch in den Gebirgen sommerliche Gewitterregen immer häufiger aus, sodass nun auch in $3000 \mathrm{~m}$ Höhe die Versteppung fortschreitet.

Botanisch Interessierte würden wohl kaum auf den abgeweideten Flächen noch interessante Pflanzen vermuten, doch um den See entstand in den im Frühjahr teilweise überfluteten Randzonen eine artenreiche Hutweiden-Gesellschaft mit Cirsium esculentum, Gentiana barbata, Parnassia laxmanii, Swertia marginata und Leontopodium ochroleucum.

Meist sind die Böden sehr flachgründig. Durch die Schneeschmelze entstehen kleine Bäche. In die- 
sem humusarmen trockenen Randbereich wächst ein unauffälliger Lippenblütler, der zu den wichtigsten zentral-asiatischen Heilpflanzen gehört und längst schon von der Pharmaindustrie entdeckt wurde. Die stark nach Minze duftende Ziziphora clinopodioides hat starke antibakterielle Wirkung.

Umgeben wird das ebene Weideland von einer etwas über $4000 \mathrm{~m}$ hohen Bergkette, wo im Juli die Schneeschmelze noch andauert. Erst im Spätsommer werden dort die Schafe alles abweiden. Anfang Juli hingegen trifft man auf ein Blütenmeer seltenster Pflanzen. Den Kratzdisteln nahe steht die nach einem russischen Botaniker benannte, seltene Schmalhausenia nidulans. In den Schmelzwasserrinnen blühen üppig Allium atrosanguineum, Oxygraphis glacialis, Tulipa dasystemon sowie die im Tien Shan endemische, weiß-blühende Trollblume Trollius lilacinus.

Aus den bunten Wiesen leuchten die orangefarbigen Köpfchen von Erigeron aurantiacus. Weithin kann man die blassvioletten Massenbestände von Geranium saxatile erkennen. Nach nur wenigen Straßenkehren hinunter in das Naryn Tal verändert sich die Landschaft schlagartig. Im Übergang zur Halbwüste wachsen Dornbüsche von Caragana jubata.

\section{Botanische Eindrücke aus dem Alai}

Der nördliche, größtenteils zu Kirgistan gehörende Teil des Pamirs wird als Alai bezeichnet. Über den Pamir-Highway, eine sehr gut ausgebaute Straße, erreicht man von Osh kommend in wenigen Stunden Sary Tash, die höchstgelegene Siedlung des Landes auf 3170 m Höhe. Ein atemberaubender Blick über das breite Tal des Kyzyl suu (Rotes Wasser) auf die schneebedeckte Bergkette des Pamirs lässt fast vergessen, wie hart das Leben hier ist. Die wenigen Touristen werden in den einfachen Gästehäusern freundlich aufgenommen.

Auf den Schwemmböden des Flusses Kyzyl suu leuchten die tiefroten Blüten von Hedysarum daraut-kurganicum, weiße helmartige Blüten von Scutellaria paulsenii, in großer Anzahl Leontopodium ochroleucum und die flach an den Boden

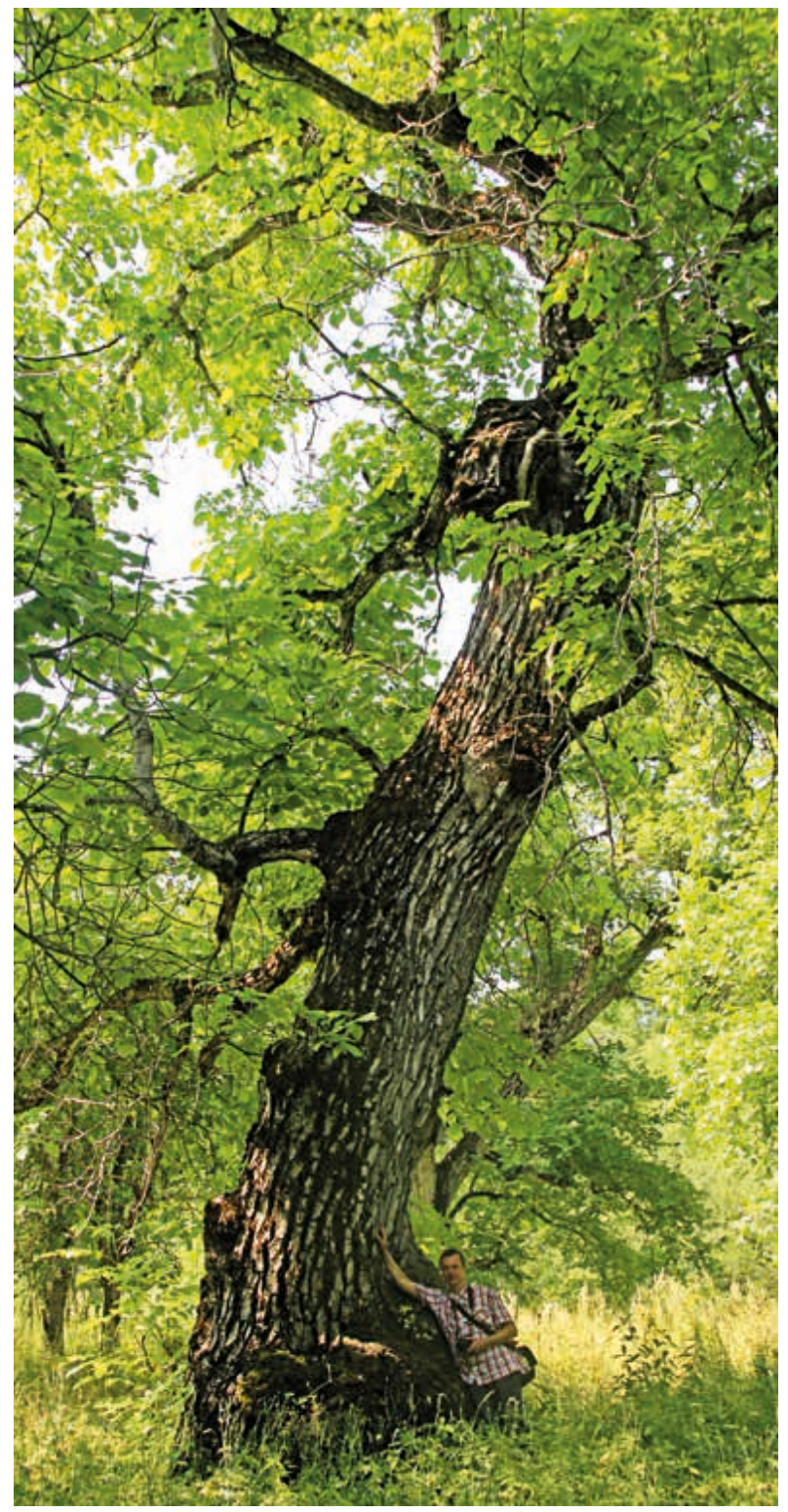

Abb. 6: Alter Walnussbaum im Biosphärenreservat Sary Tschelek.

gedrückten Rosetten von Inula rhizocephala mit großen gelben Blütenkörbchen.

Beim kleinen Weiler Kashka suu zweigt eine Schotterpiste Richtung Pik Lenin ab und führt durch eine beeindruckende Igelpolster-Steppe, geprägt von Acantholimon diapensioides-Polstern. Die sehr artenreiche Gattung Acantholimon ist in Kirgistan mit 34 Arten vertreten, die ein wesentliches Element der innerasiatischen Igelpolstergesellschaft bilden.

Wer nicht den Gipfel ersteigen möchte und sich lieber an der Pflanzenwelt am Fuße des Pik 


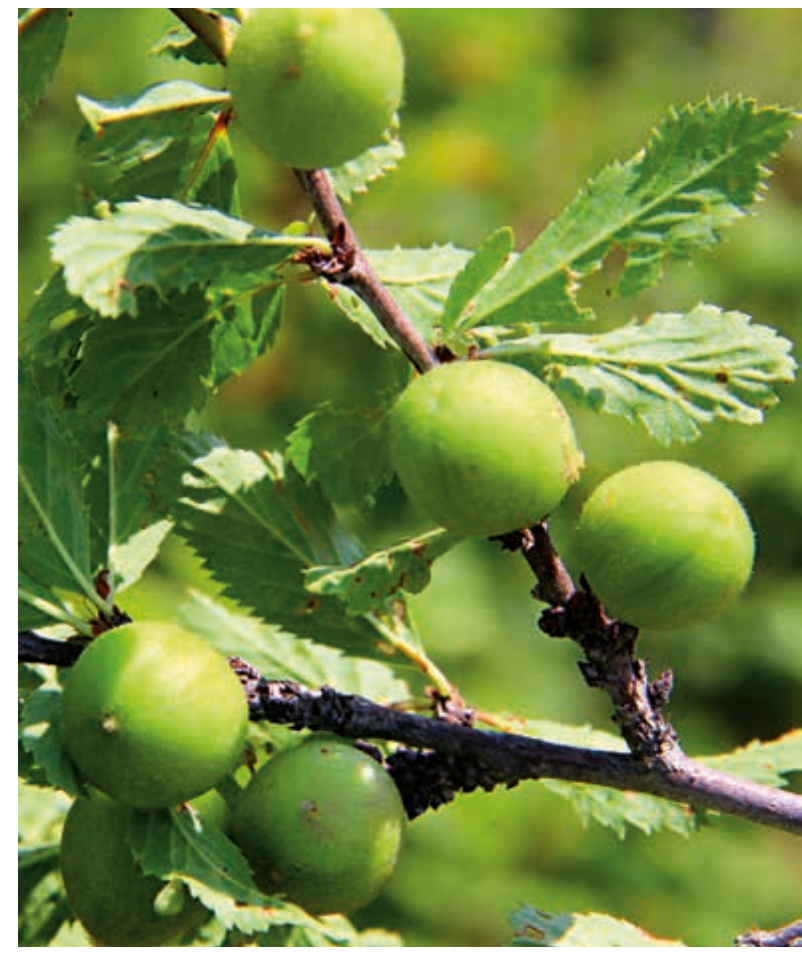

Abb. 7: Wild-Pflaume (Prunus sogdiana).

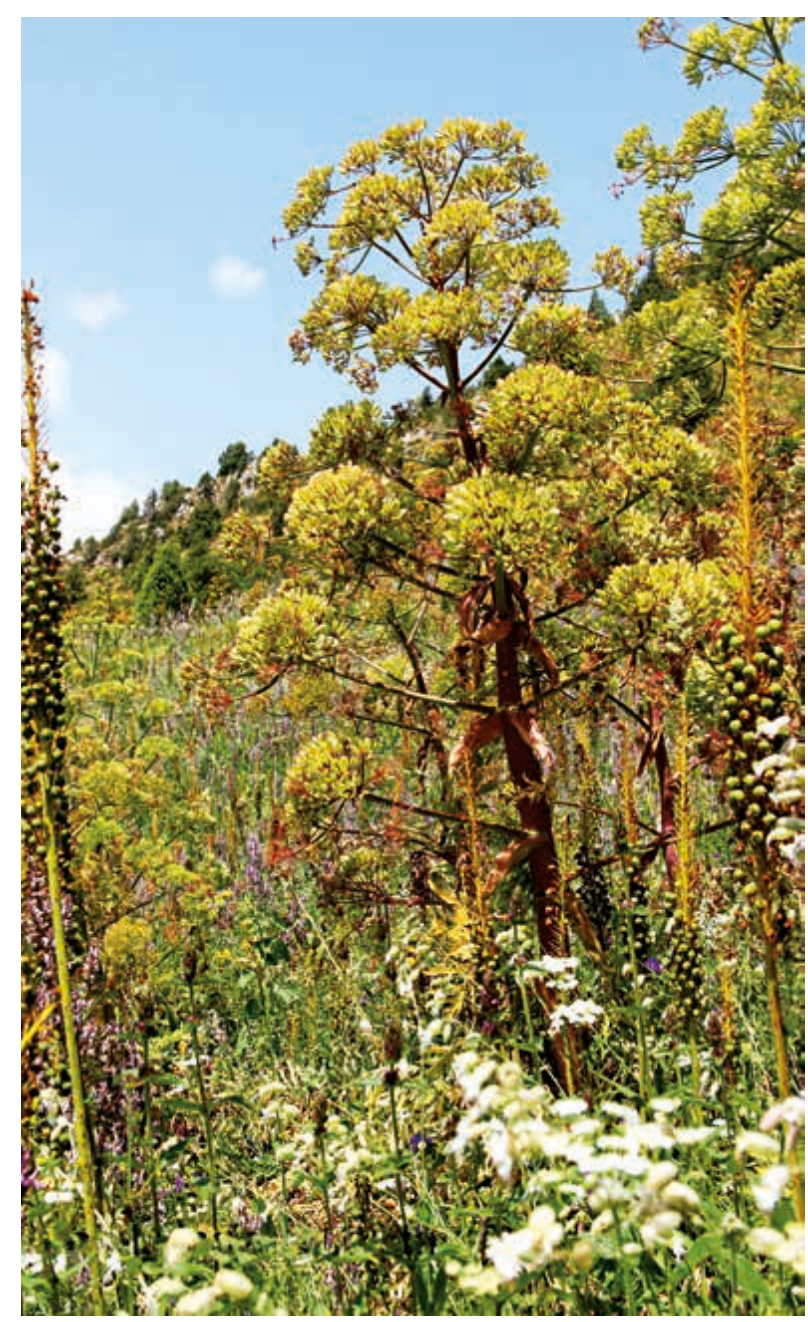

Abb. 8: Wild-Fenchel (Ferula inciso-serrata)
Lenin auf 3800 m Höhe erfreuen möchte, wird reichlich belohnt: ein weißblühender Himmelsherold (Eritrichium villosum), die nickenden Blütenglöckchen von Pulsatilla campanella, ein nur $30 \mathrm{~cm}$ hoher Rhabarber (Rheum spiciforme), das dicht filzig behaarte schneeweiß leuchtende Fingerkraut (Potentilla hololeuca), aber auch noch die auf dieser Höhe gerade erst aufblühende Tulipa dasystemon. Auf etwas humusreichen Böden blüht recht zahlreich Ligularia alpigena.

Sary Tash ist ein wichtiger Verkehrsknotenpunkt mit Verbindung nach China und über den Kysyl Art-Pass nach Berg-Badachschan (Tadschikistan). Die strategisch wichtige Verbindung wurde bereits 1932 fertiggestellt und diente im Afghanistankrieg den sowjetischen Truppen als Nachschubroute. Nur mit Ausnahmegenehmigung können Touristen die teilweise abenteuerliche Straße bis zum Grenzübergang auf $4250 \mathrm{~m}$ Höhe befahren. Es ist ein kurzer Blick in den zentralen Pamir mit ausgedehnten, lebensfeindlichen Gebirgs-Wüsten. Das windgefegte Plateau vor der tadschikischen Grenze mit den versalzenen Böden kann nur von wenigen Spezialisten bewohnt werden: Braya pamirica und Dilophia salsa ducken sich mit kleinen Rosetten und winzigen weißen Blüten in seichte Mulden, während an geschützten, wenig versalzenen Stellen die erst vor kurzem beschriebene Androsace pavloskyi sowie Chorispora elegans, Oreoblastus sabulosa und Saussurea leucophylla blühen.

\section{Tien Shan, die Himmelsberge}

Es ist nicht mehr so einsam in den Himmelsbergen! Für die Wirtschaft Kirgistan ist der Goldbergbau in der Kumtor-Mine kaum zu ersetzen. Der Gewinner ist ein kanadisches Bergbauunternehmen. Und die Umwelt? Um an den Schatz der Himmelsberge zu gelangen, wird ein ganzer Gletscher abgetragen.

Vom Südufer des Issyk Kul bei der Ortschaft Barskoon zweigt die gut ausgebaute Straße auf den Barskoon Pass ab. Am Talschluss muss ein Kontrollpunkt der Bergbaugesellschaft passiert werden, denn Freude hat hier wohl niemand mit Touristen. Die Bergbaugesellschaft will keine Besucher bei 


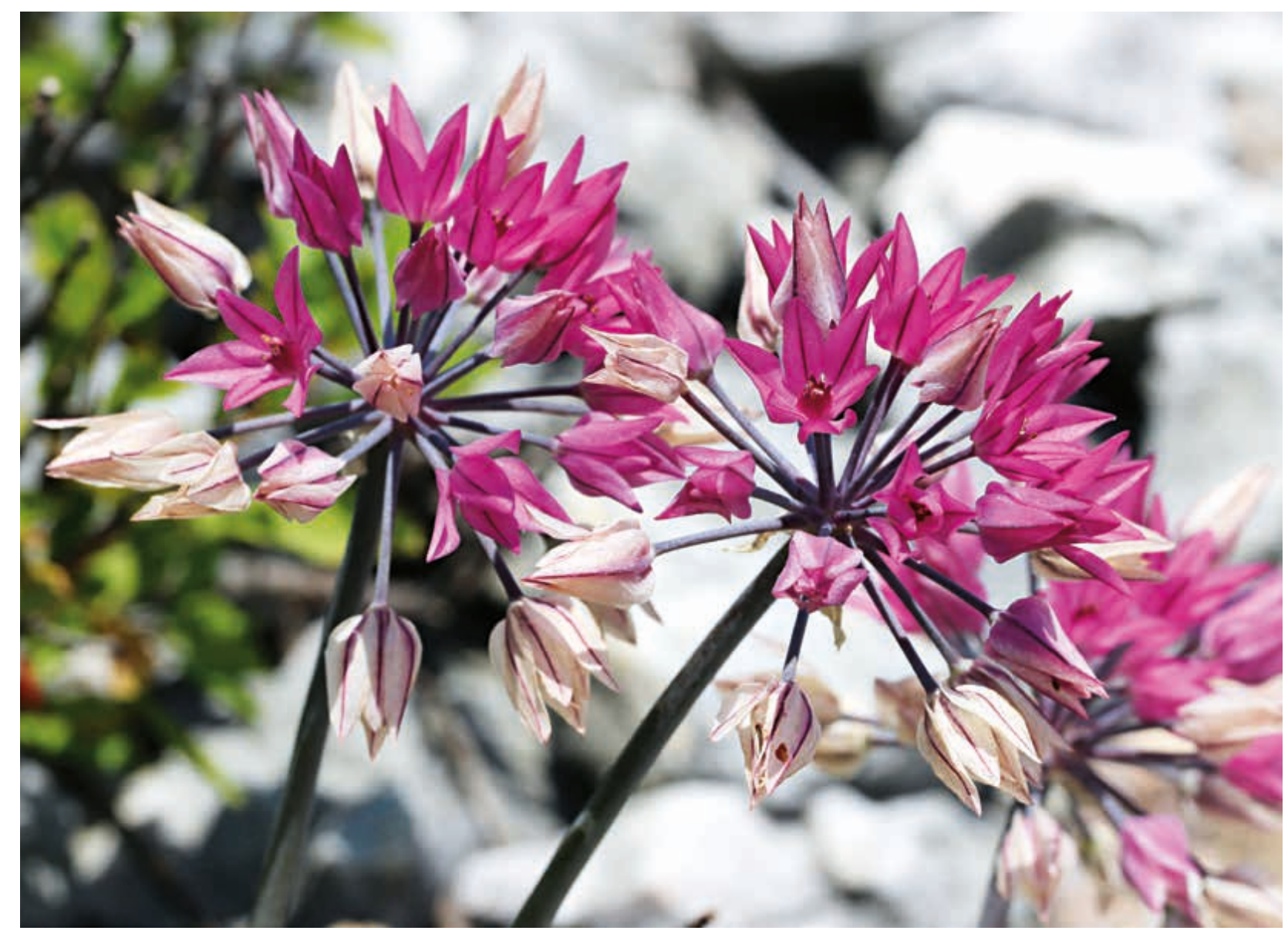

Abb. 9: Rosen-Lauch (Allium oreophilum).

der Goldmine. Eigentlich darf man entlang der Straße auch nicht anhalten, denn unentwegt donnern schwere Containerfahrzeuge über die bestens ausgebaute Straße und hüllen die Umgebung in eine dicke Staubschicht. Schon einmal stürzte ein mit giftigem Zyanid beladener Transporter in die Schlucht.

Auf den Schuttfeldern entlang der Straße gibt es auch „Goldschätze“, ein Blumenmeer u. a. mit den tiefblauen Blüten von Dracocephalum imberbe, mit orangefarbigen Blütenteppichen von Papaver croceum, zudem Erigeron heterochaeta, Tanacetum karelinii und lockere Rispen von Calamagrostis anthoxanthoides.

Am Barskoon-Pass auf 3674 m Höhe öffnet sich eine scheinbar endlose Bergtundra mit ausgedehnten Streifenböden. Für kurze Zeit tauen die Permafrostböden oberflächlich auf und die wenigen gut angepassten Pflanzen dieses Lebensraumes müssen den kurzen Sommer nützen. Gut ange- passt an die Streifenböden sind die Kissenpolster des Gelblings (Sibbaldia tetrandra) mit cremefarbenen Blüten. In den wassergefüllten Gräben blühen mit leuchtend-gelben Blüten Saxifraga hirculus und Ausläufer treibender Saxifraga macrocalyx. Dicht behaarte Rosetten von Saussurea gnaphalodes bewohnen hingegen die flachen Geröllfelder. Wo der Schnee erst vor kurzem zurückgewichen und das Geröll durchfeuchtet ist, leuchten von der Ferne schon violettrosa Massenbestände von Primula turkestanica, durchsetzt von Chorispora bungeana, Oreoblastus flabellatus, Rhodiola coccinea und Smelowskia calycina.

An den südexponierten grasigen Hängen findet man Gentiana kaufmanniana, der nur im Tien Shan und Pamir vorkommt zusammen mit Gentiana algida, dessen Verbreitungsgebiet bis zum subarktischen Nord-Amerika reicht.

Im zentralen Tien Shan, wo sich die höchsten Gipfel auftürmen, liegt einer der längsten 


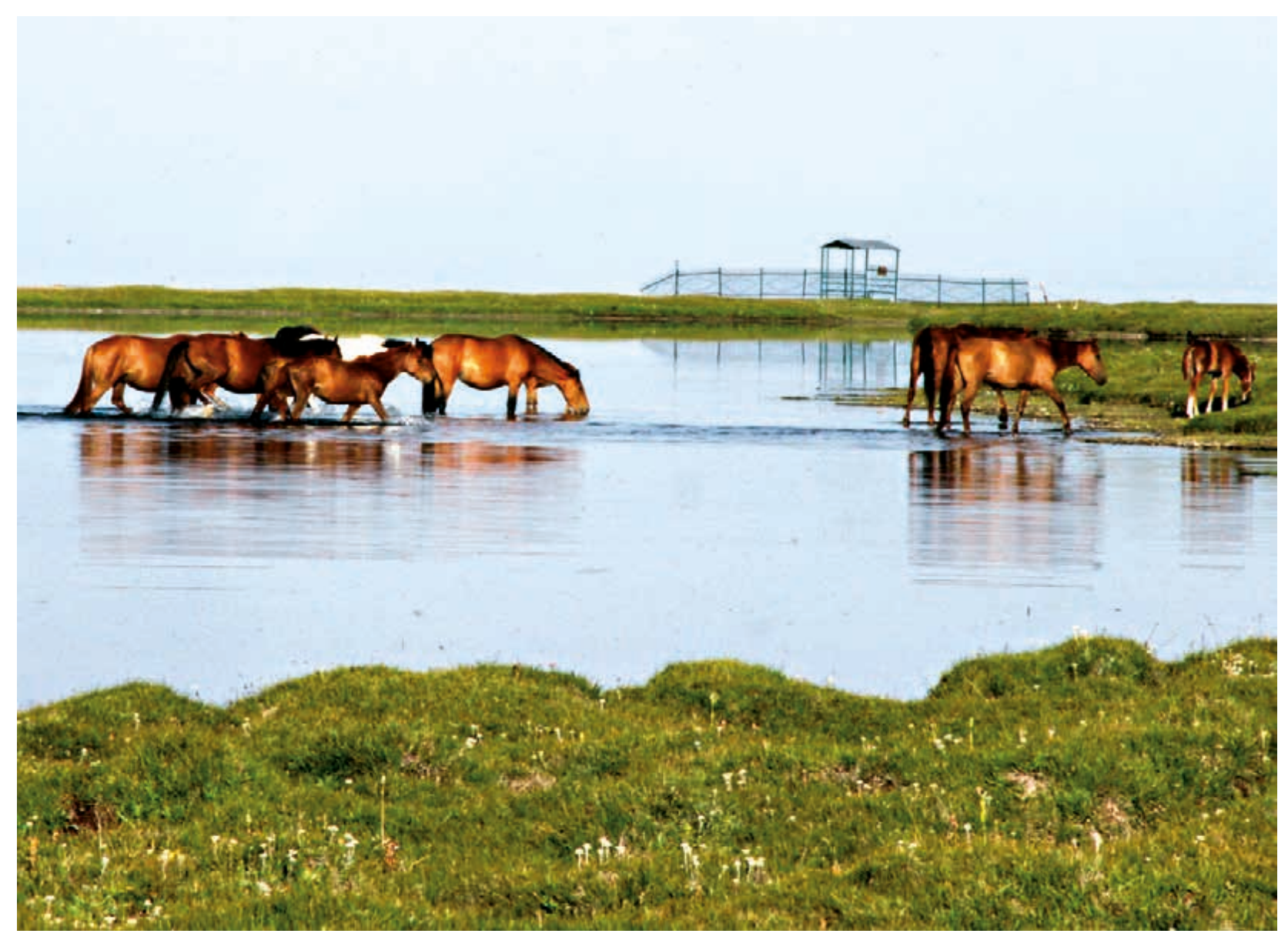

Abb. 10: Auf der Hochebene um den Song Kul.

Gletscher außerhalb des Polargebietes, nämlich der Inyltschek-Gletscher. In einem schlechten Zustand ist die Straße auf den 3817 m hohen Tschong Aschuu-Pass. In verfallenden Häusern auf der Passhöhe muss ein Straßenwärter ausharren, um die Straße offen zu halten, obwohl der Bergbau im $30 \mathrm{~km}$ entfernten Inyltschek schon lange eingestellt ist.

Zwischen Fels und Geröll an der Grenze des pflanzlichen Lebens überraschen einige Besonderheiten wie die Alpenscharte Saussurea involucrata mit bis zu $30 \mathrm{~cm}$ großen Blütenständen, die von leuchtend weißen Deckblättern geschützt werden. Dichtbehaarte Blütenkörbchen von $P y$ rethrum (Richteria) leontopodium trotzen an den Windkanten den eiskalten Stürmen. Im Felsschutt und in Felsspalten blühen zwergiges Geißblatt (Lonicera simenowii), weiter Waldheimia tridactylites, Taraxacum lilacinus und Saxifraga oppositifolia ssp. asiatica.
Im Geröll unter der Passhöhe leuchten die schneeweißen Trollius lilacinus und Callianthemum alatavicum zwischen den blauen Polstern von Dracocephalum imberbe hervor.

\section{Schlussbetrachtung}

Nach anstrengenden Tagen zwischen Steppe, Halbwüste und Hochgebirge, an Tagen tropischer Hitze im Fergana-Tal und Eiseskälte am Fuße der $7000 \mathrm{~m}$ hohen Bergriesen, übernachtet in Jurten oder bei Gastfamilien, ist die Rückkehr in unser überreguliertes Leben nicht ganz einfach. Aber die Erinnerungen bleiben wie die Sehnsucht, wieder nach Zentralasien zurückzukehren.

\section{Literatur}

Laskov, G. \& Sultanova, B. 2011: Cheklist of vascular plants of Kyrgystan. - Norrlinia 24: 1-166. (auf Russisch) Schreiber, D. 2015: Kirgistan, zu den Gipfeln von Tian Schan und Pamir. - Berlin. 